\title{
Extension of the ICRF for selected areas down to the 16th magnitude $-\|^{\star, \star \star}$
}

\author{
J. I. B. Camargo ${ }^{1,2}$, C. Ducourant ${ }^{1,2}$, R. Teixeira ${ }^{1,2}$, J.-F. Le Campion ${ }^{1}$, M. Rapaport ${ }^{1}$, and P. Benevides-Soares ${ }^{1,2}$ \\ 1 Observatoire de Bordeaux, UMR 5804, CNRS/INSU, BP 89, 33270 Floirac, France \\ 2 Instituto de Astronomia, Geofísica e Ciências Atmosféricas da Universidade de São Paulo, Caixa Postal 3386, \\ 01060-970 São Paulo SP, Brazil
}

Received 9 April 2003 / Accepted 4 June 2003

\begin{abstract}
In this paper we provide a major upgrade of the work presented in Camargo et al. (2001), aiming at the extension of the ICRF at optical wavelengths in regions of special astronomical interest, using observations from the Bordeaux and Valinhos meridian circles. Along with the new fields, the main differences, when compared to the first release, are: a much larger sky coverage, the replacement of the AC2000 by its upgraded version AC2000.2 as one of the first epoch astrometrical sources, inclusion of Tycho-2 and 2MASS ${ }^{\star \star \star}$ photometry when available, and the correction for a magnitude equation on the Valinhos right ascension system as well. The resulting catalogue contains 678828 entries with positional external precisions, on both coordinates, ranging from $50-60$ mas $(V \leq 13.5)$ to $70-140$ mas $(13.5<V \leq 16.0)$. For the proper motions, precisions range from 3 mas/year to about 15 mas/year, depending on magnitude and declination.
\end{abstract}

Key words. astrometry - reference systems

\section{Introduction}

One of the most important tasks of astrometry rests upon the adoption of a concept and the materialisation of a celestial reference system, from which one can derive the positions of heavenly bodies as well as study their variations as a function of time.

The corresponding celestial reference frame is given by a catalogue containing the coordinates of reference points along with other parameters, so that it is possible for the user to materialise the reference system at any given epoch.

Three characteristics are important for such a reference frame. They are inertiality, rigidity and accessibility. Briefly speaking, inertiality can be understood as non-rotation of the coordinate axes, rigidity relates to the agreement between the

Send offprint requests to: J. I. B. Camargo,

e-mail: camargo@obs.u-bordeaux1.fr

* Based on observations made with the CCD meridian circle at the Bordeaux Observatory, Floirac - France. Based on observations made with the CCD meridian circle at the Abrahão de Moraes Observatory, Valinhos - Brazil. Based on measurements made with the MAMA automatic measuring machine.

$\star \star$ Table 2 is only available in electronic form at the CDS via anonymous ftp to cdsarc.u-strasbg.fr $(130.79 .128 .5)$ or via http://cdsweb.u-strasbg.fr/cgi-bin/qcat?]/A+A/409/361, and Tables 1 and 3 are only available in electronic form at

http://www.edpsciences.org

$\star \star \star$ This paper was prepared before the release of the all-sky 2MASS survey. coordinate axes materialised by any subset of the reference frame, and accessibility is achieved by the appropriate availability of reference points in the sky, so that the user can use a suitable number of such points in a sky region. This work is concerned mainly with this last topic.

A major breakthrough in astronomical reference systems was achieved with the adoption by IAU, as from 1st of January 1998, of the ICRS (International Celestial Reference System) (Feissel \& Mignard 1998; Ma et al. 1998; Johnston \& de Vegt 1999), as the celestial reference system to replace the FK5 (Fricke et al. 1988). The ICRS is materialised by a set of 212 extragalactic compact radio sources listed in the ICRF (International Celestial Reference Frame) (Ma et al. 1998). The positions of these sources have been precisely determined by VLBI techniques to accuracies better than 0.5 mas.

In optical wavelengths, the ICRS is realised by the HIPPARCOS catalogue (ESA 1997) with astrometrical parameters for about 118000 objects, typically brighter than $V=$ 10.0 (completeness up to $V=8.0$ ), distributed all over the sky. This gives a star density of about 3 objects per square degree, which is insufficient to provide a desirable accessibility to the ICRS. The improvement given by the Tycho-2 catalogue notwithstanding ( $\sim 50$ stars per square degree and limiting magnitude $V=12.5$ - completeness up to $V=11.0$ ), small field astrometry has still its density needs unfulfilled.

A worldwide effort is in progress to extend the ICRF in optical wavelengths from ground-based observations, supported by the IAU Commission 8 new working-group The "Future 

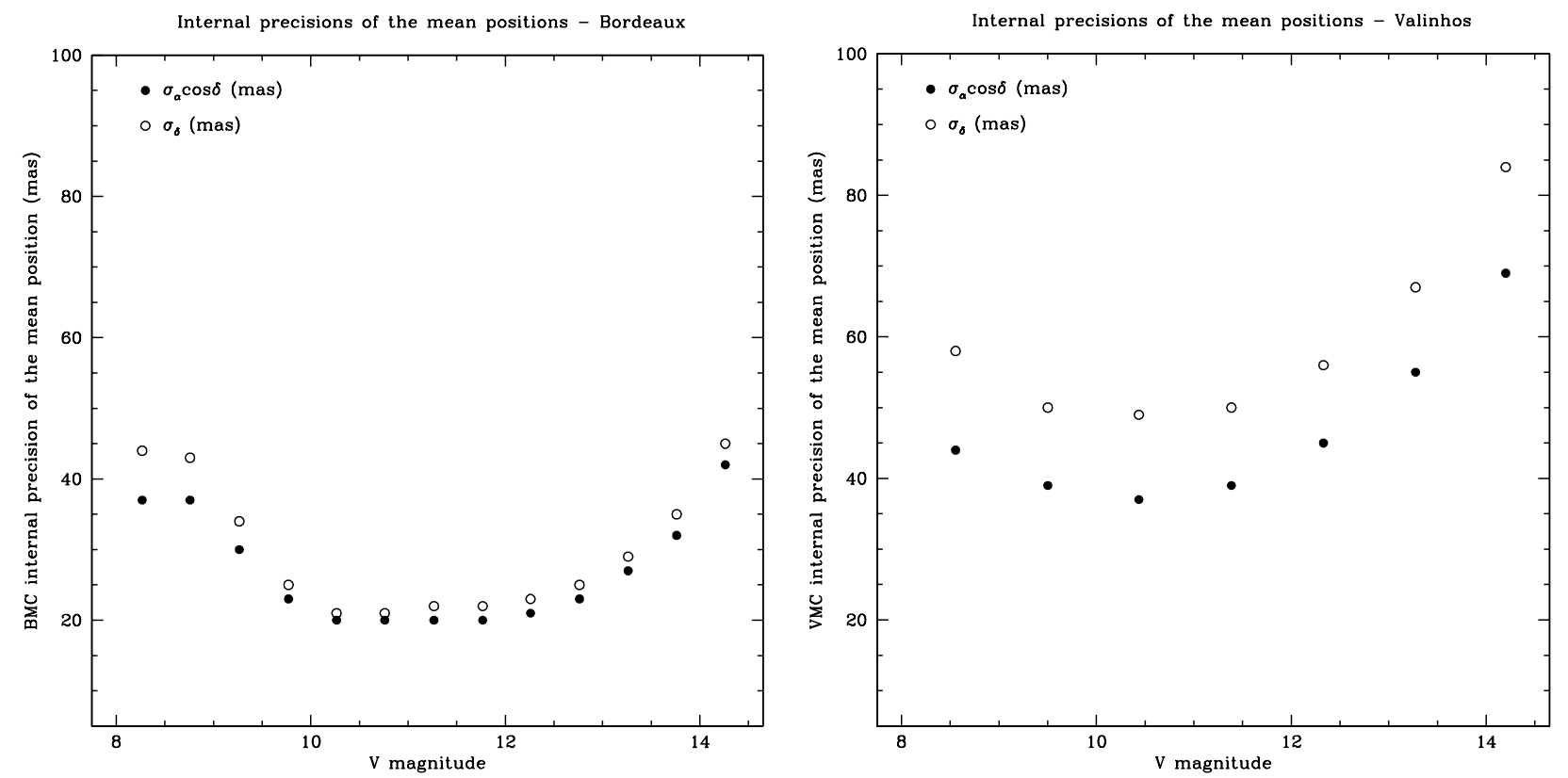

Fig. 1. Internal precisions of the mean positions of the Bordeaux and Valinhos meridian observations.

Development of Ground-Based Astrometry”. Examples of such an effort are the USNO-A2.0 catalogue (Monet et al. 1998), UCAC project (Zacharias et al. 2000), A Catalogue of Faint Reference Stars in 398 Fields of Extragalactic Radio Reference Frame Sources - ERLcat (de Vegt et al. 2001), Valinhos Meridian Circle Catalogue - VMCC (Camargo et al. 2001), and M2000 (Rapaport et al. 2001) among others.

The present work is part of this effort and upgrades its previous version, the VMCC. The main differences are: a) observational data now provides 511525 objects from the Bordeaux CCD meridian circle (hereinafter BMC) and 167303 objects from the Valinhos CCD meridian circle (hereinafter VMC) - only BMC data was retained whenever common objects between both instruments were detected. VMC data from Camargo et al. (2001), as will be discussed in Sect. 3.2, was reanalysed for this present work; b) Tycho- $2 B$ and $V$ photometry have been included in the catalogue, as well as c) $J, H, K$ photometry from 2MASS; d) correction for a magnitude equation present in the Valinhos right ascension system, and e) replacement of AC2000 by its upgraded version AC2000.2 (Urban et al. 2001) as one of the first epoch sources for proper motion measurements.

Also, in our catalogue (hereinafter BVMCcat) we provide: mean J2000 positions and respective epochs, proper motions, $V^{1}$ magnitude from the meridian observations, internal precisions for the astrometric parameters, time interval for proper motion determination, number of meridian observations as well as the involved current and first epoch sources.

\section{The meridian circles}

The main characteristics of the BMC and VMC have already been described by various papers (Viateau et al. 1999;

\footnotetext{
1 In this work, no check for variability was performed.
}

Dominici et al. 1999; Camargo et al. 2001; Rapaport et al. 2001), and details about both of them can be found in Viateau et al. (1999).

Their locations are, respectively, Floirac $(\lambda=$ $00^{\circ} 31^{\prime} 39^{\prime \prime}$ (east), $\phi=+44^{\circ} 50^{\prime} 07^{\prime \prime}$ ) in France, and Valinhos $\left(\lambda=46^{\circ} 58^{\prime} 03^{\prime \prime}\right.$ (west), $\left.\phi=-23^{\circ} 00^{\prime} 06^{\prime \prime}\right)$ in Brazil. Both instruments use CCDs operating in drift scan mode, and the bandpass, $5200 \AA$ to $6800 \AA$, makes the resulting photometric band close to the Johnson's $V$ band system (Dominici et al. 1999).

The limiting magnitude is about $V=17.0$ for the $\mathrm{BMC}$ and one magnitude brighter for the $\mathrm{VMC}$, due to the larger $\mathrm{CCD}$ of the BMC. The internal precision of the mean positions, as a function of magnitude, is shown in Fig. 1. From those panels, it is possible to notice the better performance (about 20 mas) of the BMC compared to that of the VMC. This is explained by the different size of the CCDs, allowing a longer integration time for the BMC, as well as slight differences in the reduction parameters used in each one (ponderation of observations, eliminations). It is also possible to notice a difference (about 10 mas) between the precisions of the right ascension and declination systems presented by the VMC, the origin of which is under investigation. Left and right panels of Fig. 1 are given within the optimal range of magnitude for the instrumental measurements (Viateau et al. 1999).

\section{Astrometric data}

\subsection{Current epoch observations and reduction}

Our observational data comprises 242 meridian strips (see Table 1 and Fig. 2), whose declination heights are $28^{\prime}$ for Bordeaux and $13^{\prime}$ for Valinhos, most of them centered on extragalactic radio sources, to support programs devoted to detecting their photometric variability as well as the determination of their optical position from larger telescopes. Also, strips 

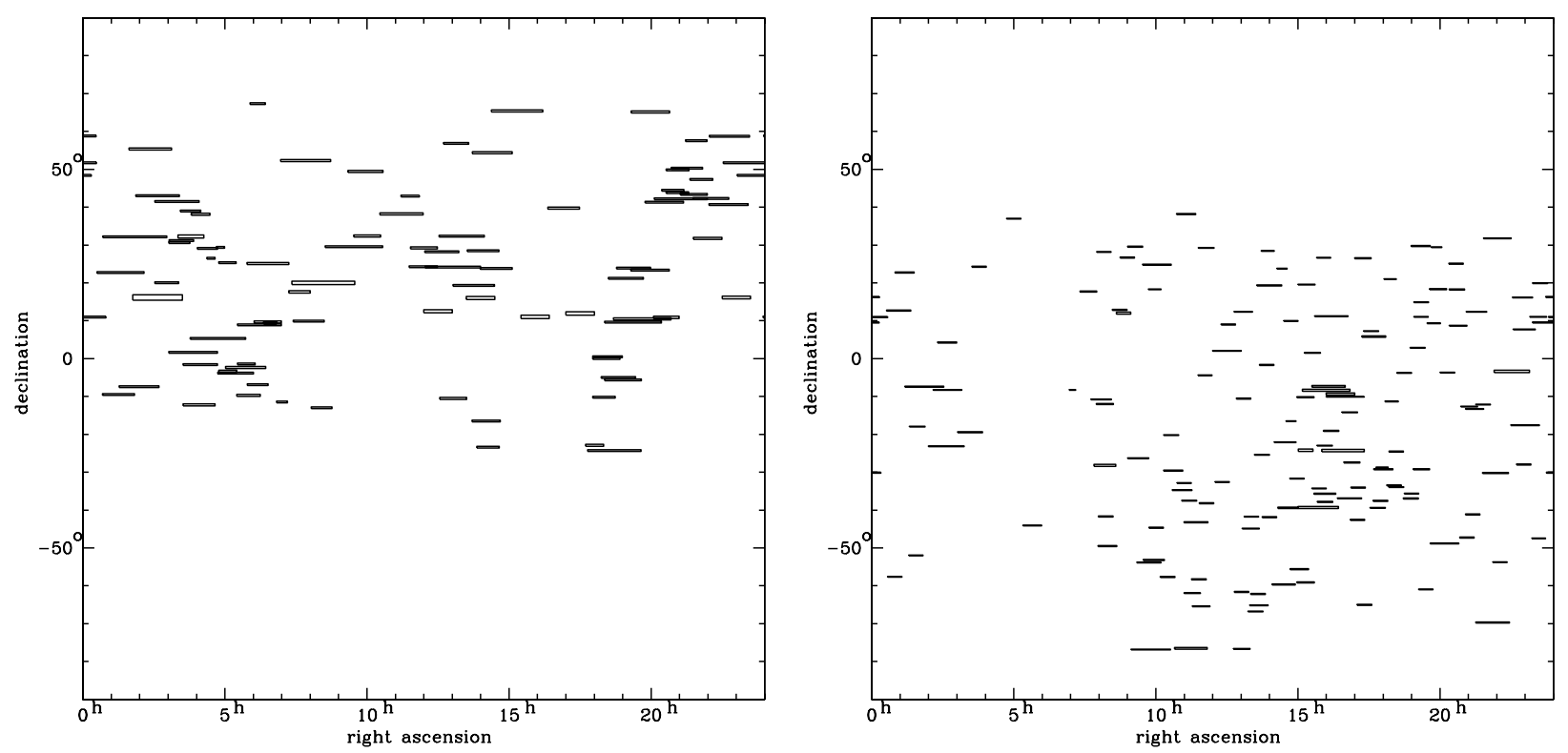

Fig. 2. Distribution of the observations in the sky as given by Bordeaux (left) and Valinhos (right). All overlapping zones were previously treated, in order to eliminate redundant entries from the BVMCcat. The adopted procedure was to retain only the BMC observation whenever an object was observed by both BMC and VMC.

containing pre-main sequence stars observed in the great southern star-forming regions (Chamaeleon, Lupus and Upper Scorpius - Ophiuchus) (Teixeira et al. 2000) and other strips containing regions of young stars, open clusters and Pluto were also included in this data set. All meridian images have been reduced (Viateau et al. 1999), for astrometry and photometry, with the Tycho-2 catalogue.

\subsection{Magnitude equation}

The presence of a magnitude equation was found on the right ascension system of the Valinhos observations, and corrected according to the curve depicted in Fig. 3, upper panel, before the determinations of the results presented here, that is, before the necessary treatment to build the BVMCcat.

This feature should be compared to the colour dependence found in Bordeaux (Rapaport et al. 2001), the cause of both effects probably being the same. It is a parallax error arising from out-of-focus images caused by the axial spread of the focal plane as a function of the wavelength. The consequent errors are essentially colour dependent. Magnitude dependence arises through the centering algorithms, which include the maximum possible number of pixels, so that bright objects will reflect the influence of the out-of-focus chromatic halo, which is not detected in the case of faint objects (Benevides-Soares 2003).

Further colour dependence effects on the meridian positions were not corrected since colour indexes were not available for all stars. No magnitude equation was found in the BMC observations.

\subsection{First and intermediate epoch material}

Positions and proper motions presented here were derived from the combination of the present epoch data (BMC and
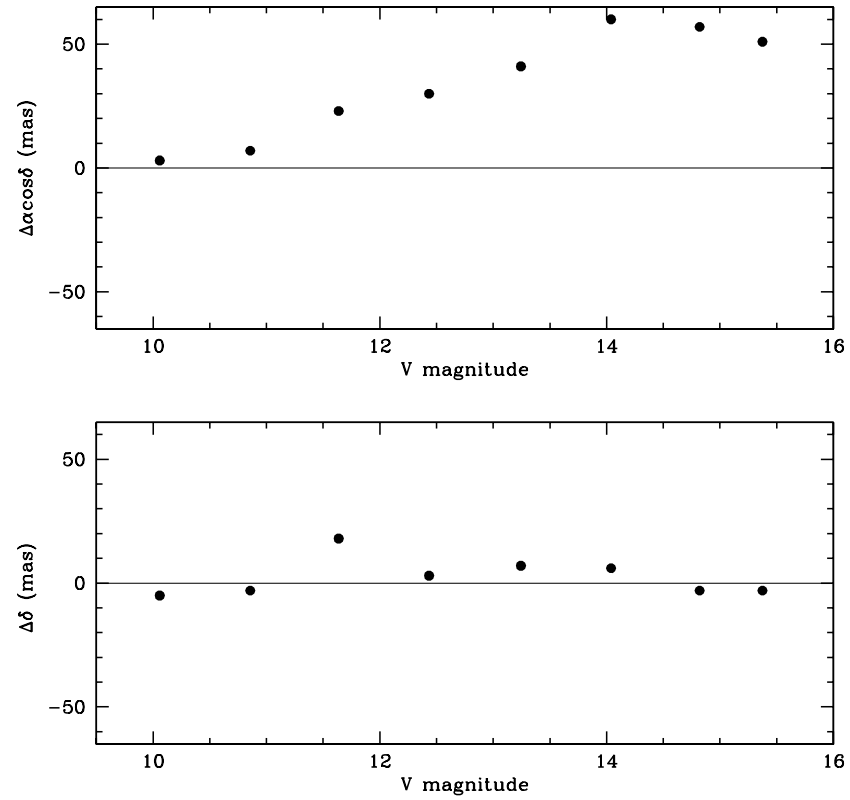

Fig. 3. Positional differences in the sense Valinhos minus Bordeaux as a function of magnitude.

VMC observations) with the aid of almost the same ancient epoch astrometric catalogues presented in Camargo et al. (2001), and following the same procedures. The main differences are the use of the AC2000.2 instead of its previous version, the AC2000 (Urban et al. 1998), and the elimination of those observational data with standard deviations larger than 250 mas in either coordinates. When a given object observed by either the BMC or the VMC had its set of counterparts identified from the first epoch material, no elimination was done on this set. This is also different from the adopted procedure in Camargo et al. (2001). In this work, AC2000.2 precisions were taken from the individual plate solutions (Urban et al. 1998). 


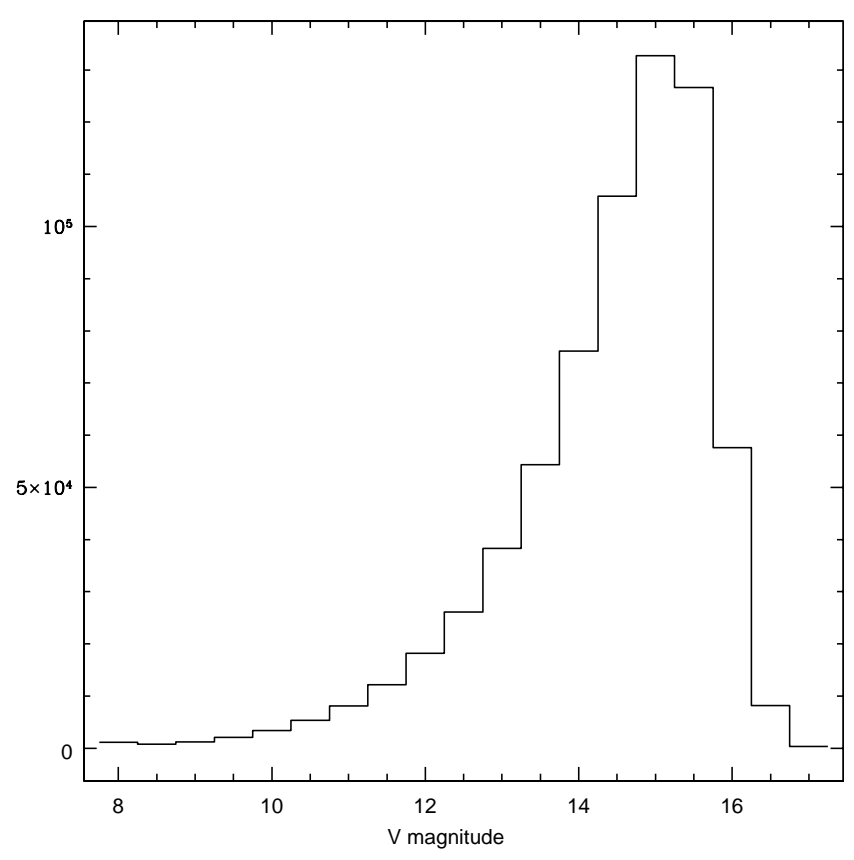

Fig. 4. Object distribution per visual magnitude in the BVMCcat.

For referencing, the other employed catalogues are presented as follows: SERC-J plates measured with the MAMA measuring machine (Guibert et al. 1983), PPM South (Bastian \& Röser 1993), PPM North (Röser \& Bastian 1998), USNOA2.0 (Monet et al. 1998), TAC-2 (Zacharias \& Zacharias 1999), and CPC-2 (Zacharias et al. 1999).

\section{The BVMCcat}

The BVMCcat contains 678828 stars brighter than $V \sim 16.0$, observed at least 3 times with either the BMC or the VMC, whose distribution as a function of magnitude can be seen in Fig. 4.

A weighted least squares procedure was used to derive positions and proper motions. The adopted weights were taken from the precisions given by the employed material. On average, these values are: 250 mas for the USNO-A2.0, 100 mas for the TAC-2, 300 mas for the AC2000.2, 270 mas for the PPM-North, 110 mas for the PPM-South, 50 mas for the CPC-2 and 300 mas for the SERC-J plates. For the meridian positions, 50 mas was used (Rapaport et al. 2001; Teixeira et al. 2000), which is a good compromise between the internal precisions of the mean positions for the Bordeaux and Valinhos instruments.

Figure 5 depicts average internal precisions of the BVMCcat for mean positions (upper panel) and proper motions (lower panel) as a function of magnitude.

The profile presented by the upper panel of Fig. 5 reflects the decreasing number of participant catalogues for the position/proper motion determination and the larger (5 times) weight given to the meridian positions as compared to the one given to those from USNO-A2.0 and the SERC-J plates. Theoretically, they should be in accordance with external verifications (presented later in the text) at least within the optimal magnitude range for the BMC and VMC astrometry (Fig. 1).
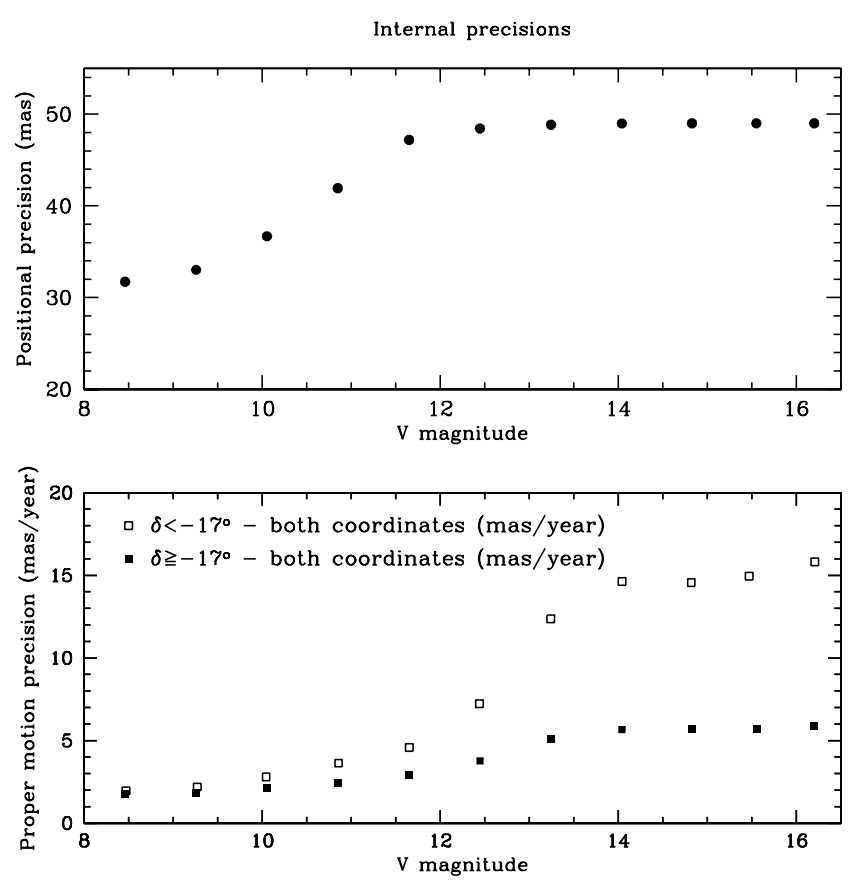

Fig. 5. Internal astrometric precisions of the BVMCcat as a function of magnitude.

To the faintest magnitudes, mean positions and their respective epochs will be close to their meridian counterparts.

The lower panel of Fig. 5 depicts a different behaviour of proper motion accuracies, given by a declination threshold of $\delta=-17^{\circ}$. As explained in Camargo et al. (2001), this is due to the USNO-A2.0, which is the only source of first epoch positions for objects fainter than $V \sim 13.0$. In USNO-A2.0 the mean epoch for objects with $\delta \leq-17^{\circ}$ is 1980 , and 1955 for those with $\delta>-17^{\circ}$.

Table 3 explains the role of each column of the BVMCcat, given in Table 2 .

\section{External verifications of the BVMCcat}

Three catalogues were employed to check the BVMCcat: the HIPPARCOS and TYCHO-2 catalogues, for the BVMCcat stars with $V \leq 12.0$, and UCAC1 (Zacharias et al. 2000), when available, for the fainter ones. All differences were obtained after transferring these reference positions, through their respective proper motions, to the epoch of the BVMCcat ones. Data derived from both meridian instruments are homogeneous after correction for the magnitude equation on the VMC right ascension system, so that no separate treatment will be provided.

\subsection{Comparison with HIPPARCOS}

The BVMCcat contains 2119 HIPPARCOS objects, out of which 1167 were selected by the criterion that eliminated those with $V<8.0$ (in order to avoid saturated observations from the meridian instruments), with HIPPARCOS MultFlag ${ }^{2}$ flag set (in order to avoid binary/multiple systems), as well as some

${ }^{2}$ HIPPARCOS' Double/Multiple Systems flag. For detailed information, please refer to ESA (1997). 

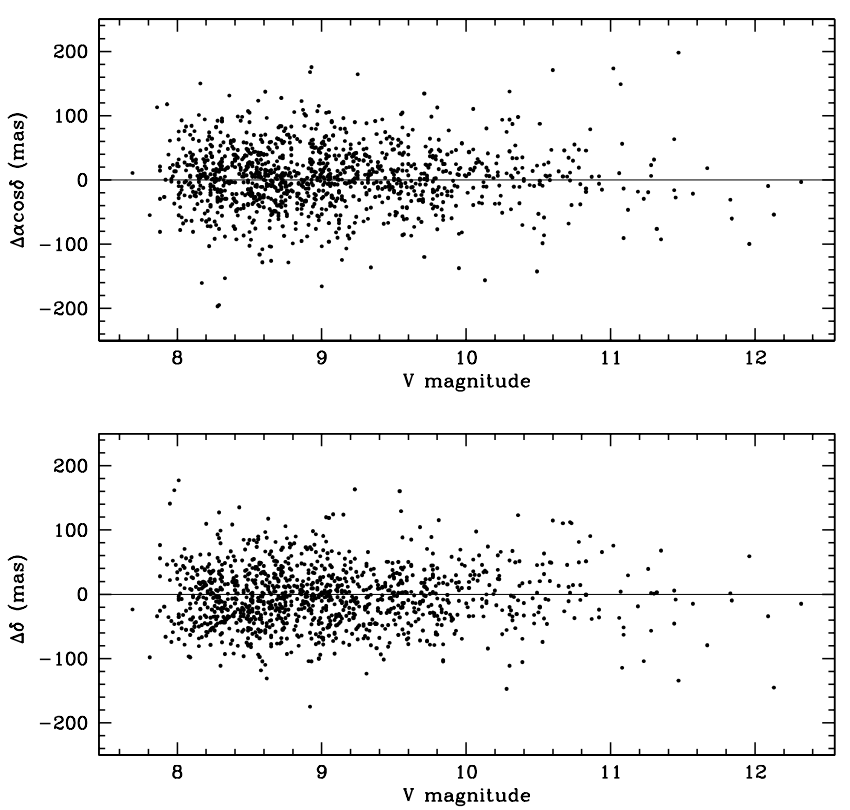

Fig. 6. Positional differences in the sense BVMCcat minus HIPPARCOS, as a function of magnitude, at the epoch of the BVMCcat. Upper panel: right ascension. Lower panel: declination.
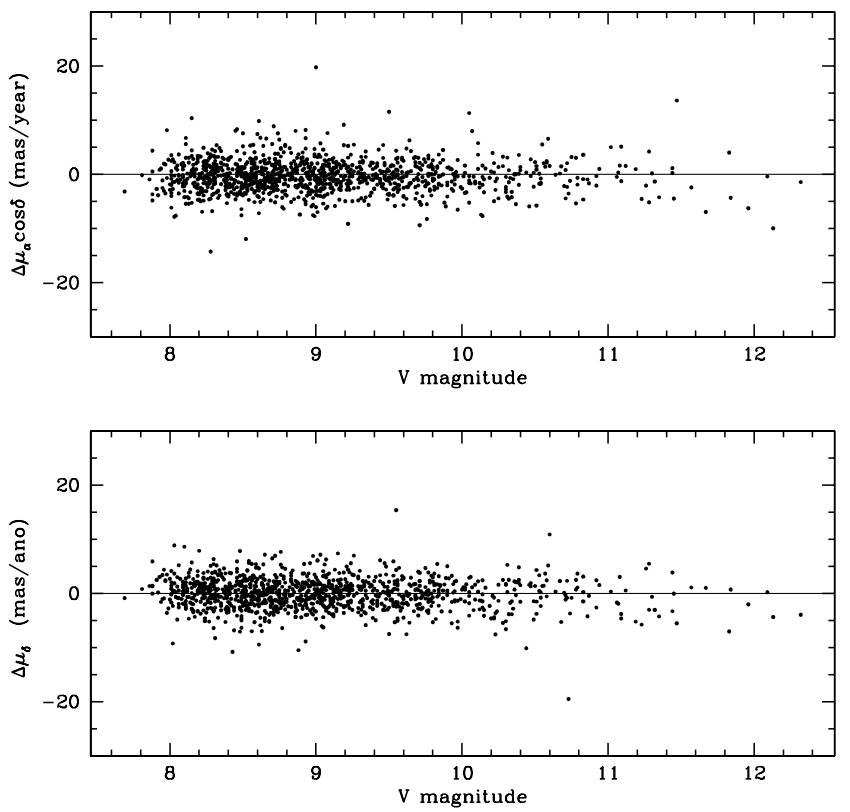

Fig. 7. Proper motion differences in the sense BVMCcat minus HIPPARCOS as a function of magnitude. Upper panel: right ascension. Lower panel: declination.

few remaining idiosyncratic points that failed to pass a $3 \sigma$ filter in position, that is, whose modulus of the positional difference were greater than 3 times the root mean square $(\mathrm{rms})$ of the differences in either coordinates.

Figures 6 and 7 depict the differences in the sense BVMCcat minus HIPPARCOS as a function of magnitude at the epoch of the BVMCcat, and Table 4 shows related quantities obtained from the rms of these differences.
Table 4. Statistical characteristics of the differences BVMCcat minus HIPPARCOS.

\begin{tabular}{lc}
\hline \hline Objects & 1167 \\
$<\Delta \alpha \cos \delta>$ & 4 \\
$<\Delta \delta>$ & -4 \\
$<\Delta \mu_{\alpha} \cos \delta>$ & -0.4 \\
$<\Delta \mu_{\delta}>$ & -0.1 \\
$\sigma_{\Delta \alpha \cos \delta}$ & 50 \\
$\sigma_{\Delta \delta}$ & 50 \\
$\sigma_{\Delta \mu_{\alpha} \cos \delta}$ & 3 \\
$\sigma_{\Delta \mu_{\delta}}$ & 3
\end{tabular}

All units are in [mas] or [mas/year]. Comparisons were done at the epoch of the BVMCcat.

The quantities presented in the aforementioned table show an overall good agreement between the BVMCcat data and that of the HIPPARCOS catalogue.

The propagated errors ${ }^{3}$ of HIPPARCOS at the epoch of the BVMCcat positions, about 10 mas, were considered negligible, so that we conclude that the BVMCcat external positional precision is about 50 mas within the magnitude interval $8.0 \leq V \leq 12.0$. The comparison of the BVMCcat proper motions with those from HIPPARCOS shows that, for objects with the AC2000.2 as the first epoch, the external error for the measurements in the BVMCcat is 3 mas/year.

\subsection{Comparison with Tycho-2}

The BVMCcat contains 45394 objects in common with Tycho-2, whose differences in positions (at the epoch of the BVMCcat) and proper motions are depicted by Figs. 8 and 9 as a function of magnitude, after a $3 \sigma$ filter in position.

Poor positional history within the employed catalogues and possible cross-identification difficulties contribute to the largest differences depicted in Fig. 9.

In Table 5, a more detailed comparison of position is shown, where a $3 \sigma$ filter within each magnitude bin was applied. For the same objects presented in the aforementioned table, related quantities for the rms of the differences in proper motions are given in Table 6.

As in the comparison with HIPPARCOS, an overall good agreement between the positions is shown by Table 5, which corroborates the value of 50 mas for the BVMCcat external positional precision (lines 6 and 7 of Table 5) when $8.0 \leq V \leq$ 11.5. External precision was obtained by subtracting from the $\sigma_{(\Delta \alpha \cos \delta, \Delta \delta)}$ values, as evaluated by the rms of the differences, the formal positional error of the Tycho-2 positions at the comparison epoch, as calculated from Høg et al. (2000).

For $V>11.5$, one can notice a degradation of the external precision of the BVMCcat, which may result partly from Tycho-2 underestimated errors in its faintest branch, as pointed out in Rapaport et al. (2001) and supported later in the text (Sect. 5.3), by the comparison BVMCcat and UCAC1.

\footnotetext{
3 According to relations 1.5.23, from Sect. 1.5 - "Transformation of Astrometric Data and Associated Error Propagation". For detailed information, please refer to ESA (1997).
} 
Table 5. Statistical properties of the positional differences BVMCcat minus Tycho-2.

\begin{tabular}{lcccccccc}
\hline \hline & \multicolumn{1}{c}{$V$ magnitude } \\
\cline { 2 - 9 } & $8.0-8.5$ & $8.5-9.0$ & $9.0-9.5$ & $9.5-10.0$ & $10.0-10.5$ & $10.5-11.0$ & $11.0-11.5$ & $11.5-12.0$ \\
\hline Objects & 571 & 963 & 1534 & 2456 & 3875 & 6113 & 9466 & 10491 \\
$\sigma_{\Delta \alpha \cos \delta}$ & 65 & 56 & 47 & 47 & 48 & 60 & 89 & 125 \\
$\sigma_{\Delta \delta}$ & 65 & 53 & 48 & 47 & 49 & 61 & 81 & 112 \\
$<\Delta \alpha \cos \delta>$ & 1 & 6 & 2 & 0 & -1 & -2 & -3 & -2 \\
$<\Delta \delta>$ & 1 & -1 & -1 & -3 & 1 & 2 & 1 & 2 \\
External precision in $\alpha \cos \delta$ & 64 & 54 & 44 & 44 & 37 & 52 & 56 & 103 \\
External precision in $\delta$ & 64 & 51 & 45 & 44 & 38 & 52 & 43 & 87 \\
\hline
\end{tabular}

All astrometric units are in [mas]. External precisions are calculated by subtracting from the $\sigma_{(\Delta \alpha \cos \delta, \Delta \delta)}$ values the respective propagated Tycho-2 error at the BVMCcat epoch.

Table 6. Statistical properties of the proper motion differences BVMCcat minus Tycho-2.

\begin{tabular}{lcccccccc}
\hline \hline & \multicolumn{7}{c}{$V$ magnitude } \\
\cline { 2 - 8 } & $8.0-8.5$ & $8.5-9.0$ & $9.0-9.5$ & $9.5-10.0$ & $10.0-10.5$ & $10.5-11.0$ & $11.0-11.5$ & $11.5-12.0$ \\
\hline$\sigma_{\Delta \mu_{\alpha} \cos \delta}$ & 3 & 3 & 2 & 2 & 2 & 2 & 2 & 3 \\
$\sigma_{\Delta \mu_{\delta}}$ & 3 & 3 & 2 & 2 & 2 & 2 & 2 & 2 \\
$\left\langle\Delta \mu_{\alpha} \cos \delta>\right.$ & -0.2 & -0.3 & -0.4 & -0.2 & -0.1 & -0.1 & -0.2 & -0.2 \\
$\left\langle\Delta \mu_{\delta}\right\rangle$ & 0.3 & 0.1 & 0.2 & 0.0 & 0.1 & 0.1 & 0.1 & 0.1 \\
\hline
\end{tabular}

All astrometric units are in [mas/year]. The same objects from Table 5 are used.
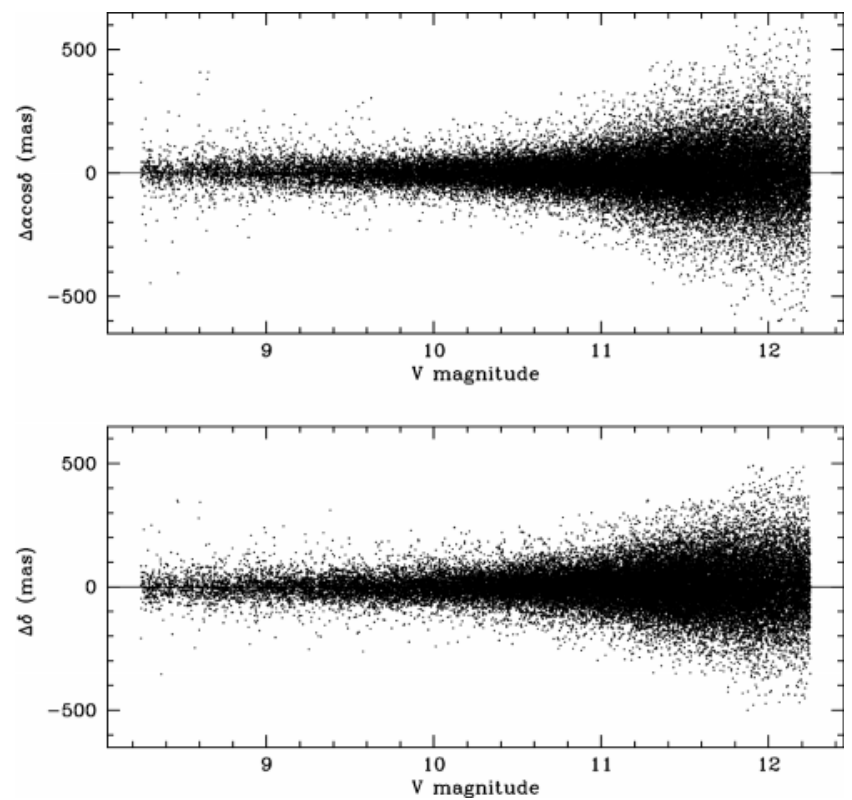

Fig. 8. Positional differences in the sense BVMCcat minus Tycho-2, as a function of magnitude, at the epoch of the BVMCcat. Upper panel: right ascension. Lower panel: declination.

From Table 6, we evaluate the BVMCcat external errors on proper motions as $\sim 2$ mas/year within the interval $8.0 \leq V \leq 12.0$.

\subsection{Comparison with UCAC1}

The UCAC1 catalogue was employed to probe the BVMCcat results on fainter magnitudes, aiming at the objects fainter than
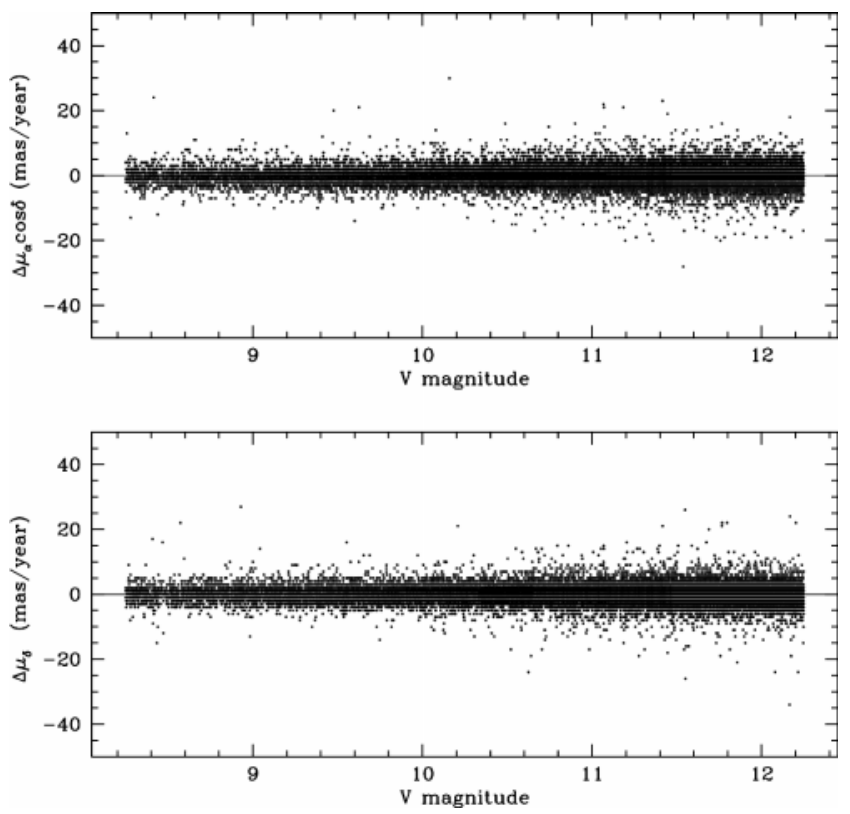

Fig. 9. Proper motion differences in the sense BVMCcat minus Tycho-2 as a function of magnitude. Upper panel: right ascension. Lower panel: declination.

$V=12.0$. We should stress that, in this verification, only the data derived from the VMC was employed, that is, the predominantly southern declinations, since UCAC1 is only available in that hemisphere.

Table 7 provides a detailed view of this comparison. In it, as in Table 5, all differences in position were binned in intervals of $0.5 \mathrm{mag}$, and those above a $3 \sigma$ filter in the respective bin were eliminated. 
Table 7. Statistical properties of the positional differences BVMCcat minus UCAC1.

\begin{tabular}{lcccccccccc}
\hline \hline & \multicolumn{10}{c}{$V$ magnitude } \\
\cline { 2 - 10 } & $11.0-11.5$ & $11.5-12.0$ & $12.0-12.5$ & $12.5-13.0$ & $13.0-13.5$ & $13.5-14.0$ & $14.0-14.5$ & $14.5-15.0$ & $15.0-15.5$ & $15.5-16.0$ \\
\hline Objects & 1963 & 3043 & 4436 & 6143 & 8767 & 12200 & 16847 & 21224 & 20154 & 6878 \\
$\sigma_{\Delta \alpha \cos \delta}$ & 49 & 52 & 55 & 58 & 65 & 71 & 81 & 100 & 129 & 141 \\
$\sigma_{\Delta \delta}$ & 53 & 58 & 62 & 68 & 73 & 80 & 92 & 112 & 145 & 154 \\
$<\Delta \alpha \cos \delta>$ & -4 & -6 & -3 & 2 & 3 & -6 & -4 & -2 & 1 & 2 \\
$<\Delta \delta>$ & -11 & -11 & -11 & -9 & -7 & -6 & -6 & -6 & -5 & -9 \\
External precision in $\alpha \cos \delta$ & 46 & 49 & 52 & 54 & 61 & 67 & 76 & 95 & 108 & 133 \\
External precision in $\delta$ & 51 & 55 & 59 & 65 & 70 & 77 & 89 & 108 & 141 & 147 \\
\hline
\end{tabular}

All astrometric units are in [mas]. External precisions are calculated by subtracting from the $\sigma_{(\Delta \alpha \cos \delta, \Delta \delta)}$ values the respective propagated UCAC 1 error at the BVMCcat epoch.

Table 8. Statistical properties of the proper motion differences BVMCcat minus UCAC1.

\begin{tabular}{lcccccccccc}
\hline \hline & \multicolumn{10}{c}{$V$ magnitude } \\
\cline { 2 - 10 } & $11.0-11.5$ & $11.5-12.0$ & $12.0-12.5$ & $12.5-13.0$ & $13.0-13.5$ & $13.5-14.0$ & $14.0-14.5$ & $14.5-15.0$ & $15.0-15.5$ & $15.5-16.0$ \\
\hline$\sigma_{\Delta \mu_{\alpha} \cos \delta}$ & 5 & 7 & 7 & 6 & 7 & 5 & 5 & 6 & 8 & 8 \\
$\sigma_{\Delta \mu_{\delta}}$ & 6 & 8 & 6 & 7 & 8 & 6 & 6 & 7 & 8 & 9 \\
$<\Delta \mu_{\alpha} \cos \delta>$ & -0.2 & 0.0 & -0.1 & 0.0 & 0.1 & -0.4 & -0.2 & -0.1 & 0.1 & 0.1 \\
$\left\langle\Delta \mu_{\delta}\right\rangle$ & 0.5 & 0.6 & 0.1 & -0.2 & -0.3 & -0.3 & -0.4 & -0.4 & -0.4 & -0.6 \\
\hline
\end{tabular}

All astrometric units are in [mas/year]. The same objects from Table 7 are used.

External precision is evaluated to 50-60 mas up to $V=$ 13.5, well within the BMC and VMC optimal magnitude interval for astrometry (Fig. 1). This external precision reaches 140 mas for the fainter objects $(V \geq 15.0)$.

The external precisions presented for the magnitude interval $11.5 \leq V \leq 12.0$, when compared to those in the same interval of Table 5, support a possible underestimation of the Tycho-2 errors for right ascensions within this magnitude interval.

To the same objects presented in Table 7, the rms of the differences in proper motions (Table 8) is 7 mas/year for both coordinates (11.0 $\leq V \leq 16.0)$, and no systematic deviation was detected. Such an estimation, however, reflects that both BVMCcat and UCAC1 use the same first epoch positions (USNO-A2.0) to derive most of these quantities.

\section{Conclusions}

The BVMCcat extends the ICRF to the optical domain in zones of special astronomical interest, and the overall agreement between the BVMCcat data and that of well-known astrometric material enables it to serve as a reference catalogue for small field astrometry as well as to provide kinematical parameters for astrophysical studies within the limits of ground-based astrometry.

The collaboration of northern (Bordeaux) and southern (Valinhos) Observatories allowed a more efficient sky coverage and a larger number of entries for the BVMCcat, as compared to our previous work (Camargo et al. 2001).

The external comparisons showed that no significant systematic trend was found either in positions or proper motions. The external errors of the BVMCcat are 50-60 mas for positions on both coordinates when $V \leq 13.5$ and 70-140 mas for fainter magnitudes $(13.5 \leq V<16.0)$. For proper motions, external errors are 3 mas/year when an AC2000.2 position is present as first epoch $(V \lesssim 13.0)$ reaching probably $15 \mathrm{mas} / \mathrm{year}$ for the faintest objects at southern declinations, as suggested by the internal error degradation (Fig. 5, lower panel).

Acknowledgements. The authors wish to express their thanks to the computer service of IAG/USP (Department of Astronomy) and of the Bordeaux Observatory, for their valuable help and patience during data-processing. The authors are also indebted to the observers of both Brazilian and French meridian circles. J. I. B. Camargo expresses his thanks to Dr. C. L. Dal Ri Barbosa and Dr. G. M. Tanco, for their help with the 2MASS data. The authors are thankful to Dr. G. Daigne, Dr. C. Soubiran and J. P. Périé for suggestions and contributions. A partial financial support from FAPESP, CAPES, PRONEX and CNRS is gratefully acknowledged. This work has made use of the NASA's Astrophysics Data System Abstract Service. This publication makes use of data products from the Two Micron All Sky Survey, which is a joint project of the University of Massachusetts and the Infrared Processing and Analysis Center/California Institute of Technology, funded by the National Aeronautics and Space Administration and the National Science Foundation. This research has made use of the SIMBAD database, operated at CDS, Strasbourg, France. The authors express their thanks to the referee, Dr. C. Ma, for fruitful suggestions and corrections. J. I. B. Camargo is supported by CNPq - Brazil.

\section{References}

Bastian, U., \& Röser, S. 1993, The PPM-South Catalogue (Heidelberg: Spektrum, Akademischer Verlag)

Benevides-Soares, P. 2003, private communication

Camargo, J., Teixeira, R., Benevides-Soares, P., et al. 2001, A\&A, 375,308 
de Vegt, C., Hindsley, R., Zacharias, N., et al. 2001, AJ, 121, 2815

Dominici, T., Teixeira, R., Horvath, J., Medina Tanco, G., \& Benevides-Soares, P. 1999, A\&AS, 136, 261

ESA. 1997, The HIPPARCOS and Tycho Catalogues, ESA SP

Feissel, M., \& Mignard, F. 1998, A\&A, 331, L33

Fricke, W., Schwan, H., Lederle, T., et al. 1988, Fifth Fundamental Catalogue - Basic Fundamental Stars (Veröffentlichungen des Astronomisches Rechen-Institut, Heidelberg), 32

Guibert, J., Charvin, P., \& Stoclet, P. 1983, in Proc. of the 78th Colloq. of the IAU, 165

Høg, E., Fabricius, C., Makarov, V., et al. 2000, A\&A, 355, L27

Johnston, K., \& de Vegt, C. 1999, ARA\&A, 37, 97

Ma, C., Arias, E., Eubanks, T., et al. 1998, AJ, 116, 516
Monet, D., Bird, A., Canzian, B., et al. 1998, in USNOA-V2.0. A Catalog of Astrometric Standards

Rapaport, M., Le Campion, J.-F., Soubiran, C., et al. 2001, A\&A, 376, 325

Röser, S., \& Bastian, U. 1998, A\&AS, 74, 449

Teixeira, R., Ducourant, C., Sartori, M., et al. 2000, A\&A, 361, 1143

Urban, S., Corbin, T., Wycoff, G., et al. 1998, AJ, 115, 1212

Urban, S., Corbin, T., Wycoff, G. et al. 2001, AAS, 1991, 2904

Viateau, B., Réquième, Y., Le Campion, J., et al. 1999, A\&AS, 134, 173

Zacharias, N., Urban, S., Zacharias, M., et al. 2000, AJ, 120, 2131

Zacharias, N., \& Zacharias, M. 1999, AJ, 118, 2503

Zacharias, N., Zacharias, M., \& de Vegt, C. 1999, AJ, 117, 2895 


\section{Online Material}


J. I. B. Camargo et al.: Extension of the ICRF for selected areas up to $V \sim 16.0$, Online Material $p 2$

Table 1. List of strips observed by the meridian circles.

\begin{tabular}{|c|c|c|c|c|c|c|}
\hline Target & \#Objects & $\frac{\sigma_{\alpha} \cos \delta \quad \sigma_{\delta}}{[\mathrm{mas}]}$ & $\frac{\alpha}{[\mathrm{J} 2000]}$ & $\frac{\delta}{[\mathrm{J} 2000]}$ & $\frac{\mathrm{mag}}{[V]}$ & Nobs \\
\hline \multicolumn{7}{|l|}{ Valinhos zones } \\
\hline NGC 7772 & 292 & 075084 & 000005.0 & +161601 & & 14 \\
\hline C 0001-302 & 220 & 081105 & 000156.5 & -300638 & & 05 \\
\hline ICRF J001031.0+105829 & 261 & 113117 & 001031.0 & +105830 & 15.4 & 10 \\
\hline ICRF J004959.4-573827 & 209 & 094101 & 004959.5 & -573827 & 18.5 & 13 \\
\hline VV96 J005334.9+124136 & 447 & 080114 & 005334.9 & +124136 & 15.7 & 10 \\
\hline ICRF J011205.8+224438 & 660 & 070091 & 011205.8 & +224439 & 20.0 & 13 \\
\hline ICRF J013305.7-520003 & 176 & 065088 & 013305.8 & -520004 & & 06 \\
\hline UV Cet & 137 & 113118 & 013627.0 & -175730 & & 05 \\
\hline ICRF J015002.6-072548 & 156 & 122118 & 015002.7 & -072548 & 15.6 & 06 \\
\hline ICRF J023951.2+041621 & 273 & 110127 & 023951.3 & +041621 & 18.5 & 08 \\
\hline ICRF J024008.1-230915 & 552 & 098104 & 024008.2 & -230916 & 16.6 & 10 \\
\hline ICRF J024104.7-081520 & 382 & 075092 & 024104.8 & -081521 & 12.3 & 06 \\
\hline J032021.2-192631 & 85 & 117143 & 032021.2 & -192631 & & 04 \\
\hline HD 23158 & 289 & 081126 & 034715.4 & +241557 & & 04 \\
\hline NGC 1778 & 104 & 137157 & 050048.1 & +365928 & & 05 \\
\hline ICRF J053850.3-440508 & 467 & 076094 & 053850.4 & -440509 & 16.5 & 08 \\
\hline NGC 2323 & 214 & 110118 & 070351.6 & -081558 & & 04 \\
\hline ICRF J073807.3+174218 & 1002 & 074099 & 073807.4 & +174219 & 16.2 & 09 \\
\hline NGC 2506 & 1062 & 076100 & 080354.6 & -104708 & & 07 \\
\hline YZ Cnc & 292 & 101117 & 080939.8 & +280820 & & 06 \\
\hline ICRF J081108.8-492943 & 1580 & 076097 & 081108.8 & -492943 & & 06 \\
\hline XY Pup & 1454 & 093097 & 081153.5 & -115905 & & 05 \\
\hline C 0810-277 & 5534 & 082094 & 081229.0 & -281235 & & 04 \\
\hline RX Pup & 819 & 068098 & 081337.8 & -414228 & & 06 \\
\hline $\mathrm{AC} \mathrm{Cnc}$ & 197 & 110127 & 084359.5 & +125040 & & 10 \\
\hline NGC 2682 & 938 & 082094 & 085156.7 & +115940 & & 07 \\
\hline WY Cnc & 308 & 073096 & 085928.6 & +264101 & & 07 \\
\hline ICRF J091552.4+293324 & 222 & 075091 & 091552.4 & +293324 & 16.4 & 05 \\
\hline ICRF J092129.3-261843 & 948 & 082097 & 092129.4 & -261834 & 18.4 & 08 \\
\hline C 0939-536 & 6004 & 086094 & 094518.7 & -535144 & & 08 \\
\hline Chamaeleon & 1094 & 090095 & 094935.8 & -764912 & & 14 \\
\hline C 0949-529 & 3789 & 078101 & 095542.5 & -531337 & & 09 \\
\hline AL Leo & 95 & 105128 & 095659.4 & +181730 & & 04 \\
\hline ICRF J100159.9-443800 & 1196 & 094099 & 100159.9 & -443801 & 17.0 & 04 \\
\hline ICRF J101353.4+244916 & 264 & 094115 & 101353.4 & +244916 & 16.6 & 10 \\
\hline IC 2581 & 4211 & 034041 & 102451.3 & -573933 & & 10 \\
\hline ICRF J103502.1-201134 & 535 & 065076 & 103502.2 & -201134 & 19.0 & 09 \\
\hline ICRF J103716.0-293402 & 1010 & 070091 & 103716.1 & -293403 & 16.5 & 11 \\
\hline TW Hya & 1041 & 041048 & 105525.1 & -344326 & & 20 \\
\hline ICRF J110331.5-325116 & 253 & 073087 & 110331.5 & -325117 & 16.3 & 08 \\
\hline
\end{tabular}


J. I. B. Camargo et al.: Extension of the ICRF for selected areas up to $V \sim 16.0$, Online Material $p 3$

Table 1. continued.

\begin{tabular}{|c|c|c|c|c|c|c|}
\hline Target & \#Objects & $\frac{\sigma_{\alpha} \cos \delta \quad \sigma_{\delta}}{[\mathrm{mas}]}$ & $\frac{\alpha}{[J 2000]}$ & $\frac{\delta}{[\mathrm{J} 2000]}$ & $\frac{\mathrm{mag}}{[V]}$ & Nobs \\
\hline ICRF J110427.3+381231 & 95 & 106139 & 110427.3 & +381232 & 12.9 & 08 \\
\hline PMN J1109-3732 & 559 & 074089 & 110948.0 & -373037 & & 06 \\
\hline Chamaeleon & 805 & 056067 & 111348.9 & -763227 & & 06 \\
\hline PMN J1117-6156 & 3972 & 070090 & 111650.4 & -615447 & & 05 \\
\hline NGC 3680 & 1410 & 035051 & 112434.9 & -431509 & & 10 \\
\hline ICRF J113143.2-581853 & 2437 & 088100 & 113143.3 & -581853 & & 10 \\
\hline SY Mus & 2481 & 106110 & 113545.1 & -652641 & & 05 \\
\hline TW Vir & 209 & 077110 & 114307.3 & -042431 & & 05 \\
\hline ICRF J114701.3-381211 & 911 & 087085 & 114701.4 & -381211 & 16.2 & 12 \\
\hline ICRF J115931.8+291443 & 228 & 091100 & 115931.8 & +291444 & 14.4 & 08 \\
\hline TXS 1217-322 & 711 & 089090 & 122002.6 & -323414 & & 08 \\
\hline ICRF J122906.6+020308 & 407 & 096095 & 122906.7 & +020309 & 12.9 & 09 \\
\hline FL Vir & 119 & 116124 & 123245.0 & +090100 & & 04 \\
\hline V850 Cen & 1889 & 098109 & 130043.1 & -613734 & & 08 \\
\hline Chamaeleon & 482 & 046044 & 130135.4 & -763933 & & 08 \\
\hline DT Vir & 136 & 120131 & 130405.8 & +122159 & & 07 \\
\hline ICRF J130533.0-103319 & 261 & 087098 & 130533.0 & -103319 & 15.2 & 09 \\
\hline V803 Cen & 1187 & 109110 & 132200.2 & -414415 & & 05 \\
\hline ICRF J132304.2-445233 & 1833 & 066080 & 132304.2 & -445234 & & 10 \\
\hline ICRF J133237.5-664650 & 5041 & 080095 & 133237.5 & -664650 & & 09 \\
\hline C $1333-619$ & 5533 & 088089 & 133539.9 & -621038 & & 11 \\
\hline ICRF J133752.4-650924 & 3474 & 084104 & 133752.4 & -650925 & & 10 \\
\hline RW Hya & 329 & 118124 & 134400.2 & -252234 & & 05 \\
\hline BH Vir & 211 & 115125 & 135407.6 & -014004 & & 05 \\
\hline ICRF J135704.4+191907 & 436 & 077094 & 135704.4 & +191907 & 16.0 & 11 \\
\hline ICRF J135900.1-415252 & 1478 & 065080 & 135900.2 & -415253 & 15.9 & 07 \\
\hline ICRF J140700.3+282714 & 325 & 098105 & 140700.4 & +282715 & 15.4 & 12 \\
\hline ICRF J142700.3+234800 & 233 & 089092 & 142700.4 & +234800 & 15.0 & 10 \\
\hline NGC 5606 & 4014 & 091093 & 143013.6 & -593933 & & 06 \\
\hline ICRF J143809.4-220454 & 960 & 080086 & 143809.5 & -220455 & 17.9 & 12 \\
\hline Lupus & 3674 & 063077 & 143837.0 & -392428 & & 23 \\
\hline ICRF J144516.4+095836 & 221 & 076092 & 144516.5 & +095836 & 17.8 & 05 \\
\hline ICRF J144553.3-162901 & 117 & 072082 & 144553.4 & -162901 & & 08 \\
\hline V822 Cen & 668 & 081101 & 145745.6 & -314011 & & 04 \\
\hline NGC 5823 & 1783 & 047056 & 150246.0 & -553449 & & 09 \\
\hline ICRF J151344.8-101200 & 454 & 110114 & 151344.8 & -101200 & 18.5 & 06 \\
\hline ICRF J151656.7+193212 & 305 & 074094 & 151656.8 & +193213 & 18.7 & 14 \\
\hline C $1511-588$ & 2175 & 084111 & 151722.8 & -590654 & & 11 \\
\hline ICRF J151741.8-242219 & 1640 & 075094 & 151741.8 & -242219 & 14.8 & 10 \\
\hline ICRF J153452.4+013104 & 345 & 074090 & 153454.5 & +013104 & 18.0 & 04 \\
\hline Lupus & 4683 & 073086 & 154246.6 & -391847 & & 17 \\
\hline
\end{tabular}


J. I. B. Camargo et al.: Extension of the ICRF for selected areas up to $V \sim 16.0$, Online Material $p 4$

Table 1. continued.

\begin{tabular}{|c|c|c|c|c|c|c|}
\hline Target & \#Objects & $\frac{\sigma_{\alpha} \cos \delta}{\sigma_{\delta}}$ & $\frac{\alpha}{[\mathrm{J} 2000]}$ & $\frac{\delta}{[\mathrm{J} 2000]}$ & $\frac{\mathrm{mag}}{[V]}$ & Nobs \\
\hline HT Lup & 799 & 088111 & 154458.0 & -341654 & & 04 \\
\hline HD 143329 & 219 & 105116 & 155422.7 & +263853 & & 08 \\
\hline HD 143006 & 537 & 112116 & 155632.7 & -225620 & & 08 \\
\hline GQ Lup & 1743 & 082105 & 155643.0 & -353953 & & 22 \\
\hline RU Lup & 271 & 103149 & 155649.4 & -374500 & & 06 \\
\hline PLUTO-97 & 2040 & 073093 & 155903.0 & -082405 & & 12 \\
\hline PLUTO-96 & 1677 & 075076 & 160233.3 & -071937 & & 27 \\
\hline НBC 630 & 706 & 089098 & 160903.8 & -190449 & & 07 \\
\hline VV96 J161033.4+111531 & 766 & 101097 & 161033.5 & +111531 & & 12 \\
\hline PLUTO-98 & 1234 & 121126 & 162917.9 & -091652 & & 11 \\
\hline$O p h+$ Upper Sco & 1419 & 053069 & 163445.9 & -241034 & & 18 \\
\hline PLUTO-99 & 364 & 106123 & 164012.3 & -100410 & & 06 \\
\hline AK Sco & 3197 & 089109 & 164848.3 & -365400 & & 12 \\
\hline HBC 652 & 274 & 114116 & 164904.8 & -141110 & & 04 \\
\hline$O p h+$ Upper Sco & 1707 & 098107 & 165336.4 & -272613 & & 04 \\
\hline HD 326823 & 602 & 111126 & 170603.4 & -423731 & & 08 \\
\hline V455 Sco & 271 & 124139 & 170652.1 & -340444 & & 04 \\
\hline Ross 868 & 138 & 124126 & 171832.3 & +262941 & & 06 \\
\hline ICRF J172341.0-650036 & 1898 & 057067 & 172341.0 & -650037 & 15.5 & 10 \\
\hline RV Oph & 369 & 113123 & 173405.0 & +071549 & & 07 \\
\hline IC 4665 & 1803 & 092110 & 173954.6 & +054816 & & 05 \\
\hline LE - bulge & 6008 & 088106 & 174841.7 & -392543 & & 08 \\
\hline LF - bulge & 6386 & 094109 & 175408.6 & -373336 & & 08 \\
\hline $\mathrm{BA}-$ bulge & 8890 & 080097 & 175751.4 & -284541 & & 08 \\
\hline $\mathrm{BB}$ - bulge & 9124 & 098107 & 180024.6 & -291321 & & 06 \\
\hline YY Her & 235 & 126138 & 181442.6 & +205858 & & 03 \\
\hline BD-11 4586 & 465 & 100107 & 181812.2 & -111813 & & 06 \\
\hline $\mathrm{BH}$ - bulge & 6333 & 093113 & 182251.5 & -333016 & & 07 \\
\hline LS - bulge & 5769 & 088101 & 182725.7 & -243341 & & 07 \\
\hline $\mathrm{BI}$ - bulge & 6061 & 089104 & 182730.8 & -335653 & & 09 \\
\hline VCLS 121 & 555 & 120124 & 184545.1 & -034705 & & 05 \\
\hline S CrA & 1669 & 079087 & 185756.4 & -365724 & & 15 \\
\hline CD- 3513069 & 1840 & 085098 & 185928.1 & -353954 & & 04 \\
\hline Gliese 748 & 2637 & 089107 & 191326.1 & +025319 & & 08 \\
\hline BF Cyg & 3781 & 096107 & 191925.7 & +294617 & & 11 \\
\hline AS 353A 7 & 1854 & 083112 & 191957.1 & +110227 & & 08 \\
\hline V1370 Aql & 1437 & 109115 & 192006.0 & +145257 & & 08 \\
\hline ICRF J192451.0-291430 & 2755 & 064089 & 192451.1 & -291430 & 18.2 & 12 \\
\hline ICRF J193006.1-605609 & 838 & 094097 & 193006.2 & -605609 & 21.5 & 11 \\
\hline OO Aql & 3872 & 110105 & 194704.2 & +091825 & & 09 \\
\hline NGC 6834 & 1962 & 059066 & 195231.8 & +292444 & & 09 \\
\hline
\end{tabular}


J. I. B. Camargo et al.: Extension of the ICRF for selected areas up to $V \sim 16.0$, Online Material $p 5$

Table 1. continued.

\begin{tabular}{|c|c|c|c|c|c|c|}
\hline Target & \#Objects & $\frac{\sigma_{\alpha} \cos \delta \quad \sigma_{\delta}}{[\mathrm{mas}]}$ & $\frac{\alpha}{[\mathrm{J} 2000]}$ & $\frac{\delta}{[\mathrm{J} 2000]}$ & $\frac{\mathrm{mag}}{[V]}$ & Nobs \\
\hline C $1950+182$ & 3875 & 070086 & 195550.5 & +181949 & & 14 \\
\hline ICRF J200925.3-484953 & 1485 & 070100 & 200925.4 & -484954 & 13.4 & 10 \\
\hline V794 Aql & 132 & 116132 & 201549.0 & -033925 & & 04 \\
\hline HD 340611 & 2144 & 079097 & 203405.4 & +250350 & & 13 \\
\hline W Del & 959 & 105117 & 203527.0 & +181553 & & 04 \\
\hline ER Del & 1852 & 080090 & 203827.0 & +084139 & & 11 \\
\hline ICRF J205616.3-471447 & 715 & 088096 & 205616.4 & -471448 & 19.1 & 11 \\
\hline NGC 6994 & 730 & 027035 & 210124.4 & -123835 & & 09 \\
\hline J210933.1-411020 & 417 & 055072 & 210933.2 & -411021 & 21.0 & 12 \\
\hline GJ 821 & 687 & 070082 & 211200.5 & -131802 & & 15 \\
\hline W Equ & 843 & 124123 & 211610.1 & +122210 & & 06 \\
\hline ICRF J213135.2-120704 & 592 & 095094 & 213135.3 & -120705 & 16.1 & 09 \\
\hline ICRF J215705.9-694123 & 652 & 095139 & 215706.6 & -694124 & 13.8 & 06 \\
\hline ICRF J215852.0-301332 & 806 & 066091 & 215852.1 & -301332 & 13.1 & 11 \\
\hline ICRF J220314.9+314538 & 1793 & 106112 & 220315.0 & +314538 & 15.6 & 06 \\
\hline ICRF J220743.7-534633 & 456 & 089094 & 220743.7 & -534634 & 18.0 & 10 \\
\hline ICRF J221852.0-033536 & 829 & 092101 & 221852.0 & -033536 & 16.4 & 09 \\
\hline ICRF J225357.7+160853 & 387 & 069109 & 225357.7 & +160854 & 16.1 & 13 \\
\hline VV96 J225405.9-173455 & 684 & 089087 & 225405.9 & -173455 & & 10 \\
\hline ICRF J225717.3+074312 & 494 & 108117 & 225717.3 & +074312 & 16.4 & 07 \\
\hline ICRF J225805.9-275821 & 249 & 099113 & 225806.0 & -275821 & 16.8 & 08 \\
\hline ICRF J232917.7-473019 & 206 & 097099 & 232917.7 & -473019 & 16.8 & 09 \\
\hline ICRF J233040.8+110018 & 395 & 062084 & 233040.9 & +110019 & 18.1 & 12 \\
\hline EQ Peg & 102 & 121111 & 233121.6 & +195531 & & 03 \\
\hline ICRF J234636.8+093045 & 557 & 077116 & 234636.8 & +093046 & 16.0 & 12 \\
\hline \multicolumn{7}{|l|}{ Bordeaux zones } \\
\hline V633 Cas & 3095 & 097077 & 001246.2 & +584943 & & 06 \\
\hline ICRF J001031.0+105829 & 1111 & 060072 & 002133.3 & +105610 & 15.4 & 10 \\
\hline ICRF J005041.3-092905 & 1210 & 060076 & 011426.9 & -093007 & 17.4 & 09 \\
\hline ICRF J011205.8+224438 & 2345 & 053062 & 011909.1 & +224229 & 15.7 & 25 \\
\hline ICRF J011935.0+321050 & 2819 & 061067 & 014917.5 & +321027 & 16.0 & 16 \\
\hline ICRF J015002.6-072548 & 1015 & 044053 & 015849.3 & -072718 & 15.6 & 33 \\
\hline RNO 6 & 6707 & 087076 & 022148.3 & +552149 & & 07 \\
\hline ICRF J023838.9+163659 & 4492 & 063070 & 023727.5 & +162800 & 15.5 & 23 \\
\hline $0129+428$ & 5385 & 081070 & 023748.4 & +430201 & & 22 \\
\hline LkHA262 & 1253 & 074084 & 025606.6 & +200500 & & 05 \\
\hline ICRF J031948.1+413042 & 9457 & 067062 & 031848.6 & +412952 & 12.5 & 19 \\
\hline RNO 13 & 493 & 082091 & 032344.4 & +30 3837 & & 06 \\
\hline LZK 4 & 719 & 078081 & 032728.7 & +311129 & & 03 \\
\hline XY Per & 1138 & 086091 & 034700.0 & +385626 & & 06 \\
\hline IP Per & 2088 & 075081 & 034818.8 & +322301 & & 09 \\
\hline
\end{tabular}


J. I. B. Camargo et al.: Extension of the ICRF for selected areas up to $V \sim 16.0$, Online Material $p 6$

Table 1. continued.

\begin{tabular}{|c|c|c|c|c|c|c|}
\hline Target & \#Objects & $\frac{\sigma_{\alpha} \cos \delta \quad \sigma_{\delta}}{[\mathrm{mas}]}$ & $\frac{\alpha}{[\mathrm{J} 2000]}$ & $\frac{\delta}{[\mathrm{J} 2000]}$ & $\frac{\mathrm{mag}}{[V]}$ & Nobs \\
\hline $0310+0122$ & 225 & 107123 & 035305.9 & +013416 & & 04 \\
\hline ICRF J040748.4-121136 & 1101 & 059064 & 040529.1 & -121341 & 14.9 & 18 \\
\hline ICRF J033930.9-014635 & 1385 & 074087 & 040739.3 & -013336 & 18.4 & 04 \\
\hline PP 13 & 460 & 096105 & 040831.4 & +380705 & & 06 \\
\hline BP Tau & 1728 & 077083 & 042250.0 & +290642 & & 06 \\
\hline DI Tau & 290 & 085093 & 043007.4 & +263216 & & 06 \\
\hline ICRF J043311.0+052115 & 5626 & 061069 & 044546.7 & +052124 & 15.1 & 25 \\
\hline DS Tau & 1125 & 083087 & 045034.1 & +292449 & & 06 \\
\hline V836 Tau & 2070 & 076082 & 050528.7 & +252233 & & 07 \\
\hline LkHA 333 & 1983 & 068083 & 050542.5 & -031948 & & 04 \\
\hline UX Ori & 1791 & 061071 & 052227.4 & -034744 & & 06 \\
\hline PQ Ori & 2761 & 059071 & 054356.5 & -022111 & & 12 \\
\hline V523 Ori & 1638 & 081100 & 054525.2 & -012235 & & 03 \\
\hline V350 Ori & 2279 & 056066 & 054933.9 & -094335 & & 09 \\
\hline ICRF J060752.6+672055 & 592 & 107088 & 060915.4 & +672029 & 20.6 & 04 \\
\hline RNO 66 & 1822 & 063074 & 060918.0 & -065553 & & 07 \\
\hline V625 Ori & 3298 & 074080 & 061252.7 & +085643 & & 03 \\
\hline W84 & 9249 & 071081 & 063019.7 & +094026 & & 06 \\
\hline $0647+250$ & 19993 & 058057 & 063050.3 & +250418 & & 23 \\
\hline MO Mon & 4684 & 073083 & 063518.4 & +092106 & & 06 \\
\hline $\mathrm{Z} \mathrm{CMa}$ & 2420 & 068079 & 070024.6 & -112826 & & 06 \\
\hline ICRF J073807.3+174218 & 6928 & 045049 & 073724.9 & +174055 & 16.2 & 38 \\
\hline 1ES0806+524 & 1006 & 073066 & 075006.6 & +522004 & & 18 \\
\hline ICRF J075706.6+095634 & 3775 & 057060 & 075641.3 & +095548 & 15.0 & 16 \\
\hline ICRF J082057.4-125859 & 3376 & 074086 & 082341.5 & -130030 & 15.0 & 04 \\
\hline OJ 287 & 7473 & 043047 & 082718.7 & +200359 & & 53 \\
\hline ICRF J091552.4+293324 & 1633 & 065069 & 093153.0 & +293321 & 16.4 & 16 \\
\hline $1011+496$ & 1565 & 064056 & 095710.8 & +492744 & & 23 \\
\hline ICRF J095820.9+322402 & 957 & 077067 & 095925.1 & +322416 & 15.8 & 09 \\
\hline ICRF J110427.3+381231 & 1728 & 058053 & 111344.5 & +381250 & 12.9 & 54 \\
\hline $1 \mathrm{ES} 1113+432$ & 233 & 084081 & 113040.7 & +425818 & & 08 \\
\hline ICRF J115019.2+241753 & 609 & 059065 & 115916.2 & +241756 & 15.7 & 14 \\
\hline ICRF J115931.8+291443 & 459 & 064070 & 115931.6 & +291512 & 14.4 & 21 \\
\hline ICRF J123049.4+122328 & 2485 & 071072 & 123003.2 & +122808 & 12.9 & 13 \\
\hline ON231 & 404 & 094098 & 123724.1 & +281329 & & 03 \\
\hline ICRF J130533.0-103319 & 779 & 090096 & 125907.1 & -102651 & 15.2 & 06 \\
\hline $1 \mathrm{ES} 1255+244$ & 362 & 074092 & 130048.0 & +241217 & & 03 \\
\hline ICRF J125614.2+565225 & 353 & 084067 & 130706.7 & +565239 & 13.8 & 13 \\
\hline ICRF J131028.6+322043 & 1631 & 057060 & 131929.6 & +322103 & 15.2 & 19 \\
\hline ICRF J135704.4+191907 & 1712 & 060065 & 134524.4 & +191853 & 16.0 & 38 \\
\hline $1400+162$ & 2653 & 059063 & 135957.3 & +160211 & & 12 \\
\hline
\end{tabular}


J. I. B. Camargo et al.: Extension of the ICRF for selected areas up to $V \sim 16.0$, Online Material $p 7$

Table 1. continued.

\begin{tabular}{|c|c|c|c|c|c|c|}
\hline Target & \#Objects & $\frac{\sigma_{\alpha} \cos \delta \quad \sigma_{\delta}}{[\mathrm{mas}]}$ & $\frac{\alpha}{[\mathrm{J} 2000]}$ & $\frac{\delta}{[J 2000]}$ & $\frac{\mathrm{mag}}{[\mathrm{V}]}$ & Nobs \\
\hline ICRF J140700.3+282714 & 926 & 072081 & 140552.7 & +282637 & 15.4 & 09 \\
\hline RXJ1412.2 & 1327 & 070085 & 141120.8 & -163012 & & 06 \\
\hline RXJ1419.3 & 552 & 091107 & 141549.4 & -232244 & & 04 \\
\hline ICRF J141946.5+542314 & 1211 & 069058 & 142514.9 & +542253 & 15.7 & 25 \\
\hline ICRF J142700.3+234800 & 1242 & 059068 & 143331.1 & +234740 & 15.0 & 06 \\
\hline $1517+656$ & 1664 & 075054 & 151723.3 & +652440 & & 59 \\
\hline $1553+113$ & 5335 & 042050 & 155459.1 & +110815 & & 49 \\
\hline ICRF J164258.8+394836 & 2855 & 055053 & 165200.8 & +394705 & 16.0 & 39 \\
\hline $1722+119$ & 15521 & 047054 & 173003.1 & +115221 & & 66 \\
\hline LkHA 122 & 8607 & 082092 & 180050.8 & -225358 & & 07 \\
\hline BD-10 4662 & 5012 & 076088 & 182031.5 & -101119 & & 06 \\
\hline VV Ser & 5535 & 067080 & 182525.8 & +000239 & & 07 \\
\hline Coku Ser G6 & 5257 & 061072 & 182741.0 & +002929 & & 07 \\
\hline LkHA 118 & 29348 & 080092 & 184246.3 & -241840 & & 08 \\
\hline AS 310 & 13588 & 061072 & 185032.8 & -045825 & & 16 \\
\hline FH Aql & 28283 & 056067 & 190102.8 & -053650 & & 15 \\
\hline WW Vul & 15200 & 059066 & 190703.4 & +211145 & & 15 \\
\hline Par 21 & 31525 & 061071 & 192141.1 & +093748 & & 13 \\
\hline LHA 483-41 & 14181 & 080086 & 192250.6 & +235236 & & 07 \\
\hline V536 Aql & 27734 & 068080 & 194114.6 & +102934 & & 08 \\
\hline $1548 \mathrm{C} 27$ & 15796 & 064071 & 195722.8 & +232315 & & 11 \\
\hline $1 \mathrm{ES} 1959+650$ & 5259 & 078060 & 195842.6 & +650833 & & 72 \\
\hline V1685 Cyg & 10044 & 059058 & 202739.9 & +412037 & & 20 \\
\hline $2032+107$ & 7621 & 055066 & 203225.9 & +105153 & & 18 \\
\hline LkHA 168 & 7222 & 069068 & 204532.3 & +443111 & & 09 \\
\hline LkHA 191 & 9099 & 067065 & 205535.1 & +435005 & & 14 \\
\hline LkHA 321 & 4004 & 081076 & 205559.7 & +495050 & & 06 \\
\hline $\mathrm{BD}+413731$ & 10758 & 069063 & 210244.0 & +421428 & & 16 \\
\hline LkHA 324 & 9583 & 078075 & 211526.9 & +501831 & & 08 \\
\hline V1082 Cyg & 13809 & 071076 & 213036.5 & +432050 & & 06 \\
\hline LkHA 349 & 5889 & 083068 & 213522.6 & +573148 & & 10 \\
\hline $\mathrm{BD}+463471$ & 15319 & 066064 & 214604.2 & +472150 & & 08 \\
\hline ICRF J220314.9+314538 & 7586 & 052057 & 215920.6 & +314608 & 15.6 & 21 \\
\hline
\end{tabular}


Table 1. continued.

\begin{tabular}{lcccccc}
\hline \hline Target & \#Objects & $\frac{\sigma_{\alpha} \cos \delta \sigma_{\delta}}{[\mathrm{mas}]}$ & $\frac{\alpha}{[\mathrm{J} 2000]}$ & $\frac{\delta}{[\mathrm{J} 2000]}$ & $\frac{\mathrm{mag}}{[V]}$ & Nobs \\
\hline ICRF J220243.2+421639 & 10808 & 060061 & 220550.5 & +421632 & 14.7 & 33 \\
LkHA 233 & 11130 & 057057 & 224304.4 & +404146 & 18 \\
DI Cep & 9224 & 077059 & 224556.6 & +584428 & 17 \\
J2253+1615 & 4501 & 047051 & 225954.4 & +161127 & 41 \\
1ES2344+514 & 21692 & 062054 & 233024.7 & +514348 & 61 \\
BM And & 10472 & 077074 & 233936.3 & +482339 & 06 \\
\hline
\end{tabular}

Target $=$ ICRF name or other identifier of the target object; Objects = Total number of selected objects in the region: 3 or more observations in each coordinate and $\sigma_{(\alpha \cos \delta, \delta)}$ less than or equal 250 mas; $\frac{\sigma_{\alpha} \cos \delta \sigma_{\delta}}{[\operatorname{mas}]}=$ Mean, in units of milliarc seconds to both coordinates, for the positional precision of the objects computed in the 2nd column; $\frac{\alpha}{[\mathrm{J} 2000]} \mathrm{e} \frac{\delta}{[\mathrm{J} 2000]}=$ Approximate central coordinates of the strips; $V=V$ mag as given in the ICRF; Nobs = Number of observations of the target region.

Zones dimensions are typically $28^{\prime}$ in declination and $80^{\mathrm{m}}$ in right ascension to the BMC and $13^{\prime}$ in declination and $40^{\mathrm{m}}$ in right ascension to the VMC.

Table 3. Explanation of the columns in Table 2.

\begin{tabular}{|c|c|c|}
\hline Field & Unit & Explanation \\
\hline RA & [hour min sec] & right ascension, ICRS, for Epoch RA \\
\hline $\mathrm{DE}$ & ' $\quad \prime \prime]$ & declination, ICRS, for Epoch DE \\
\hline$\mu_{\alpha} \cos \delta$ & [mas/year] & proper motion in right ascension, ICRS \\
\hline$\mu_{\delta}$ & [mas/year] & proper motion in declination, ICRS \\
\hline Mag: meridian & {$[\mathrm{V}]$} & meridian circle $\mathrm{V}$ magnitude \\
\hline Mag: $B$ Tycho-2 & {$[B]$} & Tycho-2 B magnitude \\
\hline Mag: $V$ Tycho-2 & {$[V]$} & Tycho-2 V magnitude \\
\hline Mag: $J$ 2MASS & {$[J]$} & 2MASS J magnitude \\
\hline Mag: $H$ 2MASS & {$[H]$} & 2MASS H magnitude \\
\hline Mag: $K$ 2MASS & {$[K]$} & 2MASS K magnitude \\
\hline Epoch RA & [year-1900] & epoch of right ascension, minus 1900 \\
\hline Epoch DE & [year-1900] & epoch of declination, minus 1900 \\
\hline$\Delta \mathrm{T}$ & [year] & largest time interval for proper motion calculation \\
\hline$\sigma_{\alpha} \cos \delta$ & [mas] & precision in RA \\
\hline$\sigma_{\delta}$ & [mas] & precision in DE \\
\hline$\sigma_{\mu_{\alpha}} \cos \delta$ & [mas/year] & precision in $\mu_{\alpha} \cos \delta$ \\
\hline$\sigma_{\mu_{\delta}}$ & [mas/year] & precision in $\mu_{\delta}$ \\
\hline Nobs & & number of observations in RA and DE \\
\hline HIP & & HIPPARCOS number \\
\hline Tycho-2 $2^{1}$ & & Tycho-2 number \\
\hline $\mathrm{AC}$ & & AC2000.2 number \\
\hline Source $^{2}$ & {$[\mathrm{~B} / \mathrm{V}, \mathrm{A}, \mathrm{U}, \mathrm{P}, \mathrm{T}, \mathrm{C}, \mathrm{E}]$} & $\begin{array}{l}\text { employed astrometric catalogues for mean } \\
\text { position and proper motion derivation }\end{array}$ \\
\hline
\end{tabular}

${ }^{1} \mathrm{An} *$ present right after the Tycho-2 number indicates that the object is not contained in the Tycho-1.

${ }^{2} B$ to BMC or $V$ to VMC; A = AC2000.2; $\mathrm{U}=\mathrm{USNO}-\mathrm{A} 2.0 ; \mathrm{P}=\mathrm{PPM}$; $\mathrm{T}=\mathrm{TAC}-2 ; \mathrm{C}=\mathrm{CPC}-2 ; \mathrm{E}=\mathrm{ESO}$ plates. 